Meta

Journal des traducteurs

Translators' Journal

\title{
De l'idée à l'énoncé : une expérience et son exploitation pédagogique dans la formation des traducteurs
}

\section{Daniel Gile}

Volume 30, numéro 2, juin 1985

URI : https://id.erudit.org/iderudit/002910ar

DOI : https://doi.org/10.7202/002910ar

Aller au sommaire du numéro

Éditeur(s)

Les Presses de l'Université de Montréal

ISSN

0026-0452 (imprimé)

1492-1421 (numérique)

Découvrir la revue

Citer cet article

Gile, D. (1985). De l'idée à l'énoncé : une expérience et son exploitation pédagogique dans la formation des traducteurs. Meta, 30(2), 139-147. https://doi.org/10.7202/002910ar

\section{Résumé de l'article}

Une expérience pédagogique montre que la verbalisation d'une idée implique l'incorporation dans l'énoncé d'éléments informationnels étrangers au message. La présence de ces excédents informationnels a d'importantes conséquences en matière de traduction et explique en partie l'origine des doutes sur la traduisibilité. Dans la traduction non littéraire, ces obstacles ne sont pas infranchissables. 


\section{DE L'IDÉE, À L'ÉNONCÉ : \\ UNE EXPÉRIENCE ET SON \\ EXPLOITATION PÉDAGOGIQUE DANS LA FORMATION DES TRADUCTEURS}

DANIEL GILE

RÉSUMÉ

Une expérience pédagogique montre que la verbalisation d'une idée implique l'incorporation dans l'énoncé d'éléments informationnels étrangers au message. La présence de ces excédents informationnels a d'importantes conséquences en matière de traduction et explique en partie l'origine des doutes sur la traduisibilité. Dans la traduction non littéraire, ces obstacles ne sont pas infranchissables.

\section{INTRODUCTION}

Comme le remarque Mortéza Mahmoudian dans un récent ouvrage sur la linguistique, pour les linguistes contemporains, l'hypothèse de l'isomorphisme entre l'organisation du signifiant et celle du signifié est apparemment dépassée ${ }^{1}$.

L'énoncé véhicule le message par évocation partielle, et non pas par application biunivoque. D'ailleurs, comment pourrait-il en être autrement, alors que :

1. La langue se compose d'un nombre fini d'éléments, et le nombre de messages susceptibles d'être véhiculés verbalement est pratiquement infini.

2. Toute idée comporte une multitude d'aspects dont la description verbale demanderait une quantité démesurée de mots.

3. Les idées s'apparentent souvent davantage à des nébuleuses qu'à des entités bien définies aux contours précis.

À l'échelon lexical, par exemple, l'énoncé nomme fréquemment une partie ou un aspect de l'objet pour désigner le tout ${ }^{2}$ : le mot " adaptateur " explicite la seule fonction du petit appareil électronique qui permet d'alimenter un poste de radio à transistors sur le courant du secteur. Pour verbaliser tous les aspects de l'adaptateur, il faudrait parler de sa forme, son poids, sa couleur, ses spécifications techniques, celles de ses composants, sa fabrication, son prix, etc.

L'énoncé ne nomme pas tous les éléments et tous les aspects du message qu'il est destiné à véhiculer; il contient par contre toujours (à de rarissimes exceptions près) des éléments d'information étrangers à ce message. La présence de ces éléments excédentaires relève en partie du choix de l'émetteur, et en partie de contraintes linguistiques .

Cette caractéristique informationnelle du langage verbal a une grande importance en matière de communication, et notamment dans le domaine de la traduction et de l'interprétation, car elle répond à des questions de méthode et aide à dissiper certains faux problèmes qui préoccupent linguistes et philosophes.

Un modèle de l'énoncé informatif considéré comme la réunion du message, informatif ou non, d'" informations non pertinentes " et de "servitudes linguistiques" est 
présenté aux étudiants du cours d'initiation à la traduction technique japonais-français de l'INALCO ${ }^{3}$ dès qu'apparaissent les premiers obstacles méthodologiques fondamentaux, en début d'apprentissage ${ }^{4}$.

Dans un souci d'efficacité pédagogique, ce modèle n'est pas présenté de manière magistrale, mais construit conjointement par l'enseignant et les étudiants au cours d'une expérience ad hoc.

Le présent article décrit cette expérience et son exploitation pédagogique.

\section{PRÉSENTATION DE L'EXPÉRIENCE}

Dans la vie quotidienne, chacun a l'occasion d'observer par introspection l'élaboration d'un énoncé à partir d'une idée, et nombreux sont les observateurs potentiels de la construction d'une " traduction " scolaire à partir d'un énoncé dans une langue étrangère. Mais il est rare que l'on puisse assister à l'ensemble du processus menant de l'idée mère à la traduction en passant par un énoncé dans une langue intermédiaire.

L'expérience conçue à l'intention des étudiants du cours de traduction technique de l'INALCO est destinée à leur permettre d'assister et de participer à ce processus, puis à réfléchir sur leurs observations, recueillies sur l'échantillon statistique qu'ils constituent collectivement.

L'expérience s'articule en deux phases :

\section{Phase A : de l'idée à l'énoncé}

À cette phase ne participe qu'une moitié d'étudiants (groupe A); la deuxième moitié (groupe B) quitte la salle en attendant la deuxième phase.

Une idée est présentée au tableau de manière non verbale (dessin) ou partiellement verbale (dessin et mots). Les étudiants lui donnent une expression verbale écrite et remettent les énoncés ainsi constitués à l'expérimentateur.

Dans notre cours à l'INALCO, la plupart des étudiants sont français ou japonais, et chacun est prié de rédiger l'énoncé dans sa langue maternelle.

\section{Phase B : de l'original à la traduction}

Dans la quasi-totalité des cas, même si l'idée mère est très simple, les énoncés des étudiants diffèrent tous, par des détails infimes ou des éléments importants.

Trois énoncés dans la même langue sont sélectionnés par l'expérimentateur qui les reproduit au tableau après avoir effacé le schéma de la phase $\mathrm{A}$.

À l'INALCO, ce sont trois énoncés japonais qui sont sélectionnés pour la phase B, afin que la traduction se fasse vers le français, langue maternelle de la majorité des participants.

Le groupe B est rappelé et les étudiants des deux groupes sont priés de traduire les trois énoncés figurant au tableau dans la langue cible.

Cette expérience révèle aux participants les trois phénomènes suivants, dont les deux premiers sont pour eux assez frappants :

1. Une idée simple, présentée d'une manière unique, donne naissance à autant d'énoncés différents qu'il y a de verbalisateurs.

2. Les énoncés obtenus ont un tronc informationnel commun, mais diffèrent par d'autres informations qu'ils véhiculent.

3. En règle générale, tous les étudiants, et notamment les membres du groupe $\mathbf{A}$, qui connaissent l'idée mère, rédigent trois traductions différentes, correspondant chacune à un "énoncé original ". 
Le déroulement de l'expérience, les observations et les conclusions qui en sont tirées en classe peuvent être expliqués utilement par le biais de l'exposé d'une expérience réalisée récemment, à titre d'illustration.

\section{ILLUSTRATION DE LA MÉTHODE : UNE EXPÉRIENCE RÉALISÉE À L'INALCO}

L'expérience décrite ci-dessous a été réalisée à l'INALCO en novembre 1982. Le groupe A se composait de 8 Français, de 7 Japonais et de 3 étudiants dont la langue maternelle n'était ni le français ni le japonais, et le groupe B de 10 Français et de 2 étudiants non francophones. Seuls sont pris en compte les énoncés français rédigés par des Français, et les énoncés japonais rédigés par des Japonais.

Le schéma de départ proposé aux étudiants était le suivant

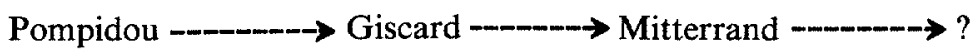

Phase A

Après avoir expliqué que le point d'interrogation était le centre de gravité de l'idée et s'être assuré que les étudiants pensaient avoir compris celle-ci, l'expérimentateur a demandé aux participants de la formuler par écrit dans leurs langues maternelles respectives.

Les énoncés recueillis étaient tous différents. Ceux des étudiants français sont reproduits ci-dessous :

1. Mitterrand a succédé à Giscard à la présidence, lequel avait succédé à Pompidou. Qui sera le président suivant?

2. En France, au gouvernement, Giscard a succédé à Pompidou, Mitterrand à Giscard. Qui lui succédera?

3. Quel sera le prochain président de la République?

4. Après Pompidou, Giscard, Mitterrand, que nous prépare l'avenir?

5. Qui pour la France demain?

6. Les derniers présidents élus furent chronologiquement Pompidou, Giscard, Mitterrand. Qui sera le prochain?

7. Peut-on savoir quel sera le prochain président d'après cette succession Pompidou Giscard Mitterrand?

8. La succession des présidents de la République est-elle celle-ci ?

Si l'on exclut de la discussion les énoncés 7 et 8 , qui témoignent de l'incompréhension de l'idée mère, il reste 6 énoncés qui véhiculent la même idée et qui sont tous différents.

Dans le contexte de la communication, et plus particulièrement de la traduction technique, qui est essentiellement informative, il est utile d'analyser ces divergences sur le plan informationnel plutôt que sur le plan linguistique.

Dans cette optique, on constate en premier lieu que le contenu informationnel de chaque énoncé diffère non seulement de celui de chacun des autres énoncés, mais aussi de celui du schéma de départ.

En effet, tous les énoncés situent le prochain président de la République dans l'avenir en conjuguant les verbes " être " et "succéder " au futur (énoncés 1, 2, 3 et 6) et en employant les mots "prochain " (énoncés 3 et 6), "avenir " (énoncé 4) et "demain " (énoncé 5). Or, le schéma de départ ne contient aucune information explicitement temporelle.

Par ailleurs, les énoncés 1, 2 et 6 situent les présidents nommés dans le passé et le présent en conjuguant au passé composé le verbe "succéder " et au passé simple le verbe "être".

En outre, les énoncés 1, 3 et 6 expliquent que les noms mentionnés désignent des "présidents"; l'énoncé 3 précise qu'il s'agit de présidents de la République ; les énoncés 
2 et 5 nomment la France, et l'énoncé 6 parle de l'élection des présidents (par opposition à une autre forme éventuelle d'accession à la présidence).

Toutes ces informations ne sont pas données par le schéma de départ.

Parallèlement à ces "gains ", on observe des "pertes" informationnelles : les énoncés 3 et 5 ne reprennent pas les noms que présente le schéma.

Ces constatations, faites à la faveur de l'observation attentive d'un phénomène quotidien sur lequel on ne se penche que rarement, suscitent des réactions de curiosité quant à l'origine de la variabilité informationnelle relevée.

\section{L'origine des "gains » informationnels}

Si l'on tente de formuler en français le schéma présenté au tableau sans y inclure les informations excédentaires relevées dans les différents énoncés reproduits plus haut, on découvre rapidement que la chose est incompatible avec la clarté de la formule et avec son acceptabilité linguistique. En fait, quel que soit le point de départ du processus de verbalisation, des contraintes linguistiques objectives d'ordre phonologique, morphologique, lexical, syntaxique et stylistique superposent des informations au message à transmettre.

On peut citer, dans le cas du français, les verbes, adjectifs, substantifs et autres mots variables qui véhiculent par le biais de règles d'accord, concrétisées par l'orthographe ou la prononciation, des éléments informationnels étrangers au message.

Dans la phrase « Je suis convaincu que ce dictionnaire rendra les plus grands services ", tirée de la préface d'un dictionnaire technique ${ }^{5}$, l'orthographe du participe passé indique que l'auteur est de sexe masculin, ce qui, compte tenu du contexte, est indubitablement une information excédentaire. D'autre part, les mots "ce", "dictionnaire " et " rendra " apparaissent au singulier, autre information excédentaire (redondante et sans intérêt) qui est véhiculée trois fois dans la même phrase.

On peut également évoquer les précisions temporelles fournies par la conjugaison des verbes, les informations géographiques transmises par le choix des mots et l'accent du locuteur, les informations sociales qui accompagnent les titres et formules d'appel.

En fait, il semble que les contraintes linguistiques soient à l'origine de la grande majorité de ces informations excédentaires, dont la présence dans l'énoncé ne résulte pas de la volonté de l'émetteur.

Il est bon de noter, toutefois, qu'il n'existe pas de " loi d'accompagnement » déterministe qui fixe les "servitudes linguistiques" (nous désignons par ce terme les informations excédentaires engendrées par des contraintes linguistiques) qui escortent un message donné. Celles-ci varient selon le point de départ du processus de verbalisation choisi par l'émetteur, selon la richesse de son vocabulaire et les autres moyens linguistiques dont il dispose au moment de l'acte illocutoire.

D'autre part, toutes les informations excédentaires ne sont pas attribuables à des contraintes linguistiques. Certains éléments sont choisis par l'émetteur pour préciser sa pensée ou pour introduire l'idée principale.

Dans certaines phrases interrogatives, c'est le rôle de la subordonnée : "Le livre que je vous ai vu lire hier est-il intéressant?"

D'autres informations non pertinentes ne sont pas sélectionnées délibérément par l'émetteur, mais sont engendrées par des habitudes verbales, des "tics", des pertes de concentration momentanées. Tel est notamment le cas de digressions et de détours que le locuteur abandonne dès que son autocontrôle se raffermit, et que le scripteur élimine lors d'une deuxième lecture de son texte.

Nous désignons collectivement les informations étrangères au message et ne résultant pas de contraintes linguistiques par le terme "informations non pertinentes". 
En règle générale, un énoncé peut être considéré comme la réunion d'un message, informatif ou non, et d'un excédent informationnel composé de "servitudes linguistiques" et d'" informations non pertinentes".

Dans l'expérience relatée ici, on constate en fait que le schéma de départ comporte aussi un excédent informationnel par rapport au message, qui est la simple interrogation sur l'identité du prochain président de la République.

Ce schéma est lui-même une représentation linguistique de l'idée (la langue en question n'étant que partiellement verbale), et obéit à des lois analogues aux lois mentionnées plus haut à propos des langues verbales naturelles.

On y relève des servitudes linguistiques, telle que la résonnance française des noms des présidents évoqués, ainsi que des informations non pertinentes, choisies pour introduire et encadrer le message, à savoir les noms mêmes et l'idée d'une progression unidirectionnelle de nature indéterminée (mais aisément interprétable grâce au bagage cognitif du lecteur français) entre ces noms.

Puisque l'idée à transmettre était la seule interrogation sur l'identité du prochain président de la République, le schéma de départ est informationnellement excédentaire par rapport au message. D'autre part, on a vu plus haut que les énoncés des étudiants sont informationnellement excédentaires par rapport au schéma. La relation excédentaire étant transitive (si B est informationnellement excédentaire par rapport à $\mathrm{A}$, et $\mathrm{C}$ est informationnellement excédentaire par rapport à $B$, alors $C$ est informationnellement excédentaire par rapport à A), les énoncés des étudiants sont tous excédentaires par rapport au message.

Par contre, ces énoncés ne présentent aucune perte informationnelle par rapport au message, puisqu'ils contiennent tous l'interrogation. Quant aux pertes informationnelles des énoncés par rapport au schéma de départ, elles sont assimilables à des pertes informationnelles dans le passage d'un énoncé linguistique à un autre énoncé linguistique, et sont examinées à ce titre dans le cadre de l'exploitation de la phase $\mathbf{B}$ de l'expérience, consacrée à la traduction.

\section{Phase B}

Les trois énoncés japonais sélectionnés pour la phase B de l'expérience réalisée en 1982 sont reproduits ci-dessous en "romaji 》 (transcription du japonais en caractères latins) à l'exception des noms propres, qui sont écrits en français :

1. Jikai kyowadaitoryo wa dare ga naru ka?

2. Pompidou, Giscard d'Estaing, Mitterrand wa rekidai furansudaitoryo no namae de aru ga, jikai wa dare ni naru ka?

3. Pompidou, Giscard Mitterrand to tsuzuita ato, tsugi no daitoryo ni wa dare ga erabareru desho $k a$ ?

Les trente étudiants des deux groupes ont traduit séparément chacune des trois phrases. En cette deuxième séance de travail de l'année universitaire, leurs "traductions", très proches du simple transcodage linguistique et marquées par la grande maladresse de la forme, sont bien représentées par le triplet ci-dessous :

1. Qui sera le prochain président de la République?

2. Les noms des présidents français successifs sont Pompidou, Giscard et Mitterrand. Mais qui sera le suivant?

3. Après que Pompidou, Giscard et Mitterrand se soient succédé, qui donc devrait être élu comme prochain président?

Priés d'expliquer en quoi se distinguaient les uns des autres les trois énoncés japonais, les étudiants ont relevé et commenté des divergences informationnelles, syntaxiques et stylistiques qu'ils attribuaient à des différences de fond. 
Exploitation de la phase $B$

Si les trois énoncés japonais engendrent trois énoncés français correspondants, c'est que chaque traducteur prend comme point de départ le vecteur linguistique du message plutôt que le message lui-même.

Pour remonter jusqu'à l'idée mère, le traducteur doit pouvoir la distinguer des informations non pertinentes et des servitudes linguistiques.

Or, si certaines contraintes linguistiques et les servitudes qui en résultent sont aisément identifiables (c'est notamment le cas des accords grammaticaux et des informations qu'ils véhiculent), d'autres le sont moins.

Dans l'énoncé 1 de la phase $\mathrm{A}$ ("Mitterrand a succédé à Giscard à la présidence, lequel avait succédé à Pompidou. Qui sera le président suivant? ") pris hors contexte et hors situation, phénomène fréquent dans la pratique de la traduction, il est impossible de déterminer la nature informationnelle du complément « à la présidence » : s'agit-il d'un élément stylistique destiné à équilibrer la phrase et relevant des servitudes linguistiques, d'une information non pertinente servant à préciser la pensée de l'auteur, ou d'un élément informationnel du message adressé à un lecteur qui ignore tout du monde politique français?

Même dans le contexte de l'expérience pédagogique qui a donné naissance à l'énoncé, la connaissance de l'idée mère et du montage expérimental ne permet pas de rattacher avec certitude cet élément à la catégorie des servitudes linguistiques ou à celle des informations non pertinentes choisies délibérément par le scripteur.

En général, la connaissance du sujet, de la situation, de l'émetteur et du destinataire permet l'identification de certaines servitudes linguistiques seulement, et le traducteur ne peut dégager complètement le message de l'excédent informationnel avec lequel il est amalgamé.

\section{L'excédent informationnel et le traducteur-récepteur}

En situation de communication verbale ordinaire, l'émetteur construit son énoncé à l'intention d'un destinataire précis, individuel ou collectif, et en fonction des connaissances et attentes présumées de celui-ci.

Dans les énoncés informatifs, les informations non pertinentes sélectionnées par l'émetteur semblent correspondre à ce que l'émetteur considère comme le minimum nécessaire à la réception du message par le destinataire ${ }^{6}$.

Or le bagage cognitif du traducteur n'est nullement assimilable à celui du destinataire, qui partage avec l'émetteur un centre d'intérêt et des connaissances communes. Il en résulte que les servitudes linguistiques et les informations non pertinentes, partiellement ou entièrement redondantes ou dénuées d'intérêt au regard du message pour le destinataire, sont fréquemment importantes, voire indispensables à la compréhension du message par le traducteur.

On constate par exemple que sans l'appui des informations non pertinentes des énoncés $1,2,3,4$ et 6 de la phase $A$, l'interrogation simple, telle que présentée dans l'énoncé 5 ("Qui pour la France demain ? "), ne peut être entièrement comprise par le traducteur.

\section{L'excédent informationnel et le traducteur-émetteur}

Si l'on pose que la fidélité est le premier devoir du traducteur, celui-ci est tenu de transmettre dans son discours l'intégralité du message original. Incapable de détacher entièrement le message des servitudes linguistiques et des informations non pertinentes, il est dans l'obligation de reprendre dans sa traduction tous les éléments de l'énoncé original susceptibles de véhiculer une partie du message. 
Si l'énoncé 4 de la phase A (" Après Pompidou, Giscard, Mitterrand, que nous réserve l'avenir ?") est présenté hors contexte et hors situation, le traducteur ne peut éliminer l'hypothèse d'un auteur politiquement engagé dont le message porte sur l'alternance "droite" " gauche ", et non pas seulement sur la simple identité personnelle des présidents de la République. Les trois noms sont donc susceptibles de porter une partie du message et ne peuvent être écartés de la traduction sans autre forme de procès.

Par contre, en situation d'interprétation, si l'interprète connaît bien les interlocuteurs et sait que l'énumération n'est qu'une information non pertinente, il ne manque pas à son devoir de fidélité s'il ne la reproduit pas et se contente de la simple interrogation.

Les principes reconnus dans les milieux professionnels veulent que l'on traduise les idées, et non pas les mots. Il n'en demeure pas moins que des contraintes cognitives empêchent en général le traducteur de remonter jusqu'à l'idée pure et l'obligent à reprendre une partie de l'excédent informationnel véhiculé par les mots, ce qui implique un certain alourdissement informationnel de la traduction par rapport à l'original.

Ces contraintes expliquent en partie la tendance de la traduction à être plus longue que l'original (on parle du "cœefficient de foisonnement " de la traduction). Cette tendance ne se concrétise d'ailleurs pas toujours de manière observable, car la langue d'arrivée peut être plus concise que la langue de départ et le traducteur plus concis que l'auteur de l'original.

\section{La traduction des informations non pertinentes}

Si l'on postule que la fidélité est acquise par la transmission du vouloir-dire de l'émetteur, on peut considérer en vertu des constatations et conclusions exposées jusqu'ici que la traduction de l'excédent informationnel est superflue.

Or, les pertes informationnelles relevées dans le passage du schéma de départ à l'énoncé (phase A de l'expérience) portent sur cet excédent informationnel, l'interrogation même étant présente dans tous les énoncés. Ces "pertes" ne méritent donc pas cette appellation et ne constituent pas une perte dans la "traduction".

Néanmoins, dans la pratique, la traduction de la majeure partie des informations non pertinentes présumées est souhaitable, et ce pour trois raisons :

1. L'impossibilité, déjà soulignée, de les distínguer du message, fait qu'il est plus prudent de les traduire.

2. Si l'on excepte les textes techniques codés (bulletins météorologiques, etc.), les télégrammes et les textes assimilés, les énoncés purement informatifs sont rares. Or, dès lors qu'un énoncé présente un aspect littéraire, fût-il minime, la forme y véhicule par définition une partie du message, et le choix des informations non pertinentes devient significatif.

3. Les informations non pertinentes choisies délibérément par l'émetteur pour préciser sa pensée peuvent être reprises par le traducteur dans le même but.

\section{Lacunes informationnelles et traduction}

Si les contraintes linguistiques superposent un excédent informationnel au message dans la verbalisation de l'idée dans la langue de première formulation, elles imposent un excédent informationnel qui peut être différent dans la traduction vers la langue cible.

Or, contrairement à l'émetteur, qui, en tant qu'auteur du message, dispose de tout le bagage cognitif requis pour satisfaire aux contraintes linguistiques liées à la verbalisation, le traducteur ne peut se fonder que sur le contenu informationnel de l'énoncé original et sur un bagage cognitif très limité. 
En français, il est souvent nécessaire, pour nommer une personne, d'employer un titre qui précise le sexe, voire le statut marital de l'intéressé (monsieur, madame ou mademoiselle). S'il est question, dans un discours japonais de "Tanakasan », l'interprète qui ne connaît pas l'intéressé a du mal à transmettre le message sous une forme linguistiquement acceptable, car le titre "san" ne véhicule pas ces informations.

De même, le traducteur francophone a souvent du mal à évoquer dans son texte un objet dont l'original japonais n'indique pas s'il est unique ou non, car le choix entre le singulier et le pluriel est obligatoire en français.

La fréquence de ce type de problème est d'autant plus grande que les langues concernées sont dissemblables, mais la difficulté n'est pas totalement absente quand la traduction porte sur deux langues proches.

Le problème des lacunes informationnelles est propre à mettre en valeur l'importance du bagage cognitif du traducteur dans un contexte plus large que celui de la seule traduction technique ou spécialisée. C'est grâce à des connaissances extra-linguistiques étendues que le traducteur est davantage en mesure de distinguer le message de l'excédent informationnel, et c'est grâce à ces connaissances qu'il est en mesure d'éviter un grand nombre d'incidents résultant des incompatibilités entre les contraintes linguistiques des deux langues en présence.

\section{CONCLUSION}

Contrairement aux exercices de version scolaire, conçus dans un but d'apprentissage, la traduction professionnelle a pour mission de communiquer un message à un destinataire. Pour cela, elle doit satisfaire à des critères de qualité sur trois plans :

- la fidélité

- la clarté

- la qualité linguistique

S'attachant à être fidèles, les néophytes cherchent à reproduire dans leur traduction la forme linguistique de l'énoncé original, ou, à défaut, le contenu informationnel de chacun de ses segments. Ils constatent rapidement que ces efforts ne peuvent aboutir qu'au détriment de la clarté et de la qualité linguistique.

Le problème de la traduisibilité, des "belles infidèles ", du "traduttore traditore " est ainsi posé.

L'expérience décrite dans le présent article explique ces oppositions sur un plan plus pratique que théorique en mettant en évidence les excédents informationnels, et notamment les servitudes linguistiques, qui contrastent d'une langue à l'autre.

Dans les textes à vocation essentiellement informative, où l'aspect littéraire (la transmission du message par le biais de la forme) s'efface en faveur de l'aspect informationnel (la transmission du message par le contenu), ces oppositions constituent un faux problème, car la fidélité consiste à transmettre le message, et n'est nullement remise en cause par l'abandon des servitudes linguistiques, qui lui sont étrangères dans l'esprit de l'émetteur.

Dans la pratique, face à un énoncé non littéraire, le traducteur se livre à une opération d'exégèse pour dégager le message, puis le restitue dans la langue cible par un énoncé clair et respectueux du génie de la langue. Le fait qu'il en résulte la disparition de certains excédents et leur remplacement par d'autres ne nuit nullement à la qualité de la traduction.

L'expérience pédagogique débouche donc sur un modèle informationnel de l'énoncé ${ }^{7}$ qui sert de base à la formulation d'un principe pratique à l'intention des élèves-traducteurs : dans la traduction d'un texte informatif, il convient d'exprimer le mes- 
sage avec ses propres mots, sans hésiter à remanier la structure linguistique de l'énoncé de départ.

Le caractère absolu de ce principe contraste avec la relativité plus subtile des textes réels, où l'informatif et le littéraire se mélangent aisément. Par ailleurs, en l'appliquant sans finesse, on risque de passer insensiblement de la traduction à l'adaptation, voire à la censure. D'où la nécessité d'un contrôle suivi de l'enseignant pour guider les efforts et modérer les ardeurs excessives dans la liberté.

L'expérience et son exploitation pédagogique sont assimilables à une impulsion donnée aux étudiants afin de les libérer de leurs habitudes scolaires de thème et de version. Les résultats obtenus sur plusieurs années donnent à penser que l'outil est efficace.

\section{Notes}

1. MAHMOUdiAN, Mortéza (1982) : la Linguistique, Paris, Seghers, pp. 207-208, 212.

2. C'est la "synecdoque" de Marianne LEDERER. Voir son article "Synecdoque et traduction", dans Études de linguistique appliquée, no 24 , octobre-décembre 1976.

3. Institut national des langues et civilisations orientales, établissement à caractère scientifique et culturel rattaché à l'université Paris III - Sorbonne nouvelle.

4. Voir Daniel GILE (1982) : "L'enseignement de la traduction japonais-français à l'INALCO ", dans Traduire, no 109, I.

5. Préface du Dictionnaire technique des fabrications mécaniques de René BOISSIER (1975) : Paris, Desforges. La préface est de A. CASTELL.

6. Cette règle, difficile à démontrer concrètement, s'explique intuitivement : tout dépassement de ce minimum est susceptible de porter atteinte à l'efficacité de l'acte de communication en délayant le message, et de démobiliser l'attention du destinataire en accumulant des informations redondantes ou étrangères à l'objet de la communication.

7. Voir Daniel GILE (1982) : "Fidélité et littéralité dans la traduction : une approche pédagogique ", dans Babel, XXVIII-1. 